\title{
Development of Dining Ontology by Using Semantic Based Information Retrieval in Cloud Platform
}

\author{
${ }^{1}$ Lakshmi Palaniappan, ${ }^{2}$ G.V.UMA \\ ${ }^{1}$ Asso. Prof. Department Of Computer Science, Ultra College Of Engineering,Madurai,India. \\ ${ }^{2}$ Prof.Department of IST,Anna university Chennai,India.
}

\begin{abstract}
Query given by the user may be keyword based or query based data. That under goes semantic search by indexing latent semantic indexing method. correct match of the data found and from Dining ontology query concept, document concept based on the search Query determine. then the adarank is used in dining ontology to get the weightage. similarity with respect to semantic obtain and evaluated. These is implemented in the cloud platform. Dining Ontology as is the expression of meaning of the content. Semantic web based the domain (Service of the restaurant is called dining). Semantic web gives instant access. Reusability is enhanced. . It focuses on the RDF, Ontology model and Web ontology language. dining ontology is developed.
\end{abstract}

Keywords: Ontology, RDF, Semantic web , keyword based, query based ,LSI

\section{Introduction}

In the proposed article a new, ontology-based approach to information retrieval (IR) is presented. The system is based on a domain knowledge representation schema in form of ontology. Ontology is a description of concepts with relation and properties to be used in knowledge engineering as a knowledge base. Ontology is used in information retrieval to retrieve more relevant information from a collection of unstructured information source. one of the approaches developed is semantic based technique to make the information retrieval more efficient. Since modelling ontologies is a tedious and costly task, it is always important to demonstrate the advantages by applying ontologies in Software Engineering.

Information retrieval is the process of retrieving relevant information from collection of unstructured information that meets the user need. The amount of information available in World Wide Web (WWW) is very large and still growing, which makes retrieval of information from WWW a tedious task. There are many search engines developed to address this problem, but most of them adopt the traditional keyword based search. Keyword based search method uses the user query to retrieve set of relevant documents from the indexed document those fit the terms given by the user

Semantic Web is an extension of current web in which information provides well-defined meaning that enables system and people for better understanding and can enable to work effectively by understanding information from different sources. The introduction of semantic web is a great leap from the existing lso has the capability to generate more meaningful information. The complete information is represented with the help of Ontology. Ontology allows knowledge to be represented as a set of concepts, properties and the relations between them . In information retrieval, the users don't search with the exact terms represented in the documents in most of the cases. Hence, relevant documents are not fetched by the keyword-based information retrieval but the semantic web makes the information retrieval more users driven than that of keyword driven. Hence it helps to retrieve more relevant documents. The semantic web makes use of various types for Ontologies for understanding the user query input in information retrieval using verb net. Domain specific ontology such as Dining is used .

The main goal of information retrieval is to retrieve relevant information based on the user request on external information source. The existing traditional keyword based search is not sufficient in most of the cases. Keyword based search process on indexed set of documents contains the keyword along with the document location. These set of keywords are searched in the document index and the document that correspond to the keywords are retrieved to the user. The semantic search has improved the information retrieval by retrieving more relevant information than that of keyword based search. The keyword based search fails in many scenarios as the user don't know the exact term what he is searching for. In the Ontology based information retrieval, the user's query input is semantically expanded by query processing with Ontologies. This semantically enhanced query searches for documents in the semantically indexed cluster of documents to retrieve the relevant information.

The semantic based information retrieval intends to search with concepts rather than with terms, which retrieves more relevant information. Various semantic based search techniques have been adopted since the evolution of semantic web. Semantic query is constructed from the user's query input by using domain Ontologies thereby retrieving related information. There are many existing methods of information retrieval, 
there is no one such technique that can be perfect for all the application need. Compared to all the models semantic method retrieve more relevant documents. So the logic-based formalisms in the context of the semantic web effort is an important factor. Activities by the W3C and others have helped by the standards like RDF or OWL. Another important factor is the flexibility of ontologies. ontologies are well-suited to combine information from various sources and infer new facts based on this. Also, the flexibility allows to extend existing ontologies very easy, thus fostering the reuse of existing work. In contrast to traditional knowledgebased approaches, e.g. formal specification languages, ontologies seem to be well suited for an evolutionary approach to the specification of requirements and domain knowledge.

Ontology is the specification of a concepts. Conceptualization is a simplified view that represent the purposes. Every ontology includes a dictionary with explanation of the terms and indications and shows relations. The ontology represents conceptual description of the specific content, to identify appropriate terms and relationship in a given knowledge domain. Ontologies show a hierarchical dependents of the terms together with descriptions, explanations and definitions. Ontologies provide a mechanism to capture knowledge about the problem domain.This deals with creation of ontology for restaurant operation, that is service of the restaurant may be called as dining, hence dining ontology created.

An ontology is a formal representation of the knowledge by a set of concepts within a domain and the relationships between those concepts. An ontology consists of individuals, classes, properties. Individuals represent objects in the domain. This deals with creation of ontology for dining .It is one of the service in restaurant, in dining ontology properties are binary relation on individuals. In this domain, dining ontology properties such as is a relationship is created.

\section{Related Work}

In the papers referred information retrieval is analyzed and semantic based information retrieval is the best analyzed in the paper. In another base paper information retrieval is obtained without symmantic approach

Base paper - Analysis have been made and semantic based information retrieval is the best method as analysed. another base paper information retrieval only obtained

1. Volume 3, Issue 10, October 2013 ISSN: 2277 128X International Journal of Advanced Research in Computer Science and Software Engineering -Analysis

2. Ontology-based Information Retrieval

Proposed - Based on the related work semantically based information retrival by key word search and query search of the domain dinning ontology is obtained In base paper IR obtained and analysed as semantic method is best but this is implemented in our work as semantic based IR and this is implemented in CLOUD platform.so it is semantic based IR from dining ontology is developed and implemented in cloud platform

\section{Description of The Domain Ontology}

A representative part of the ontology is the service of the Restaurant as DINING ontology. It involves address, contact detail of the restaurant and about catering details, that is beverages available with cost to the customers, beer, wine with price. Whether home delivery available, self service needed. . This ontology give details about cuisine, whether it is coffee shop cuisine, wine bar cuisine, icecream parlor cuisine, Indian cuisine, vegetarian cuisine and also about the Atmosphere, whether it is business \&casual atmosphere, dance floor available. dish details can be viewed and facility available as business dining ,children \&baby facility available, baby chair, baby laundry, children meal, children game area.

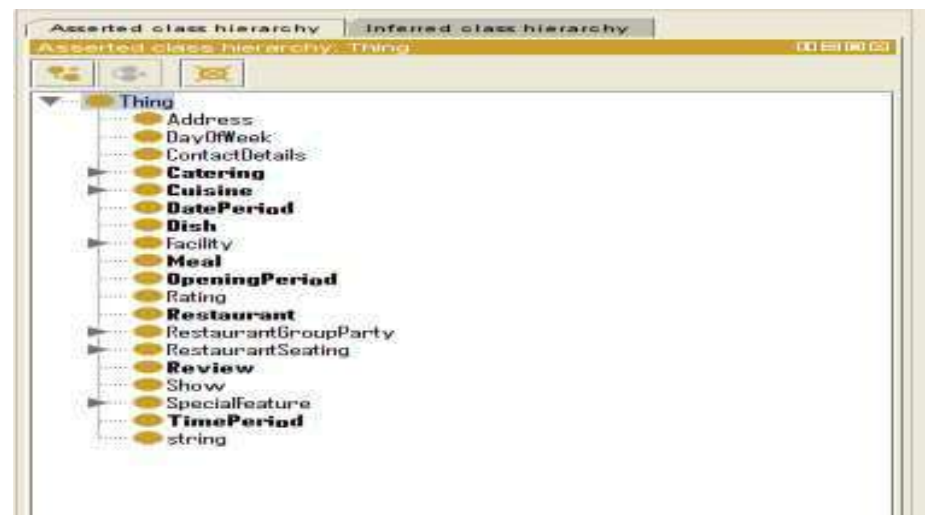




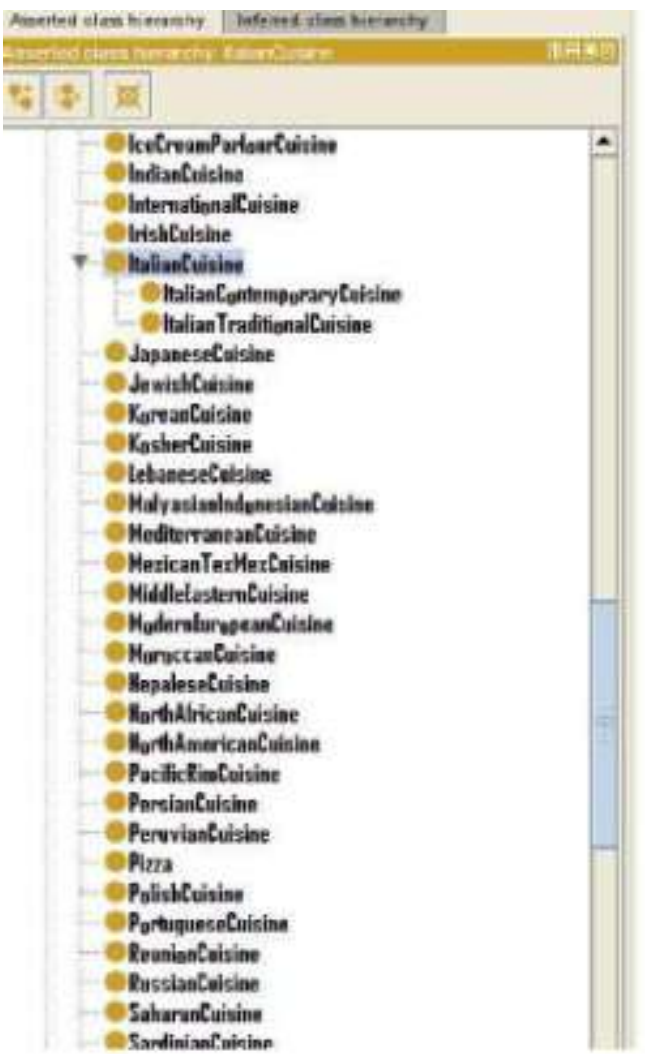

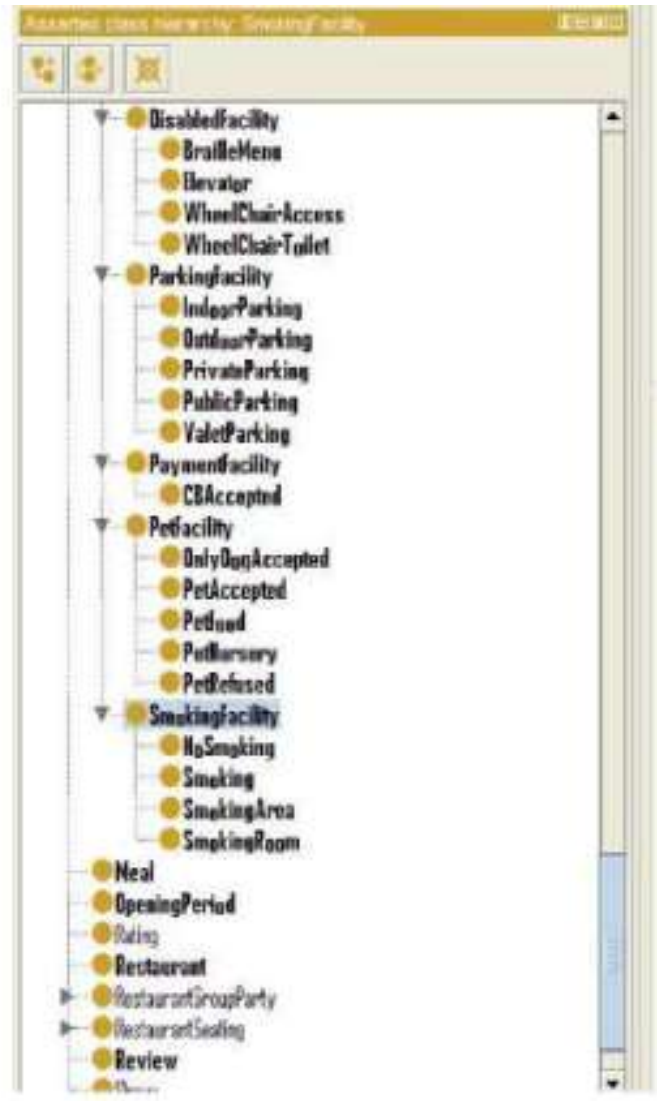

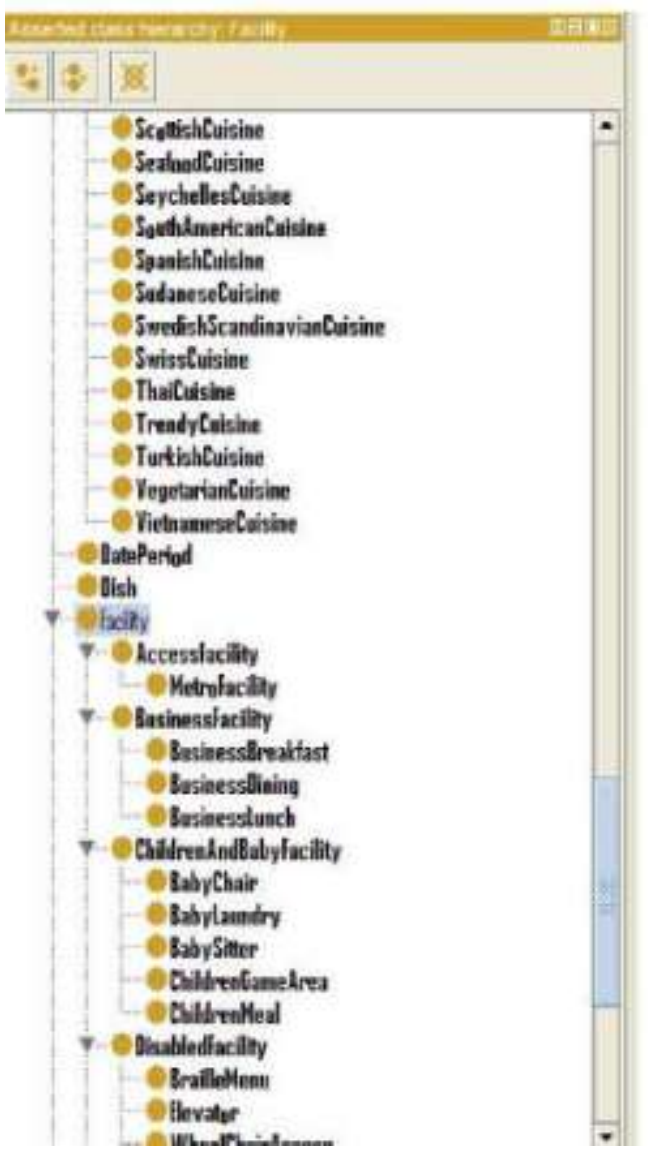

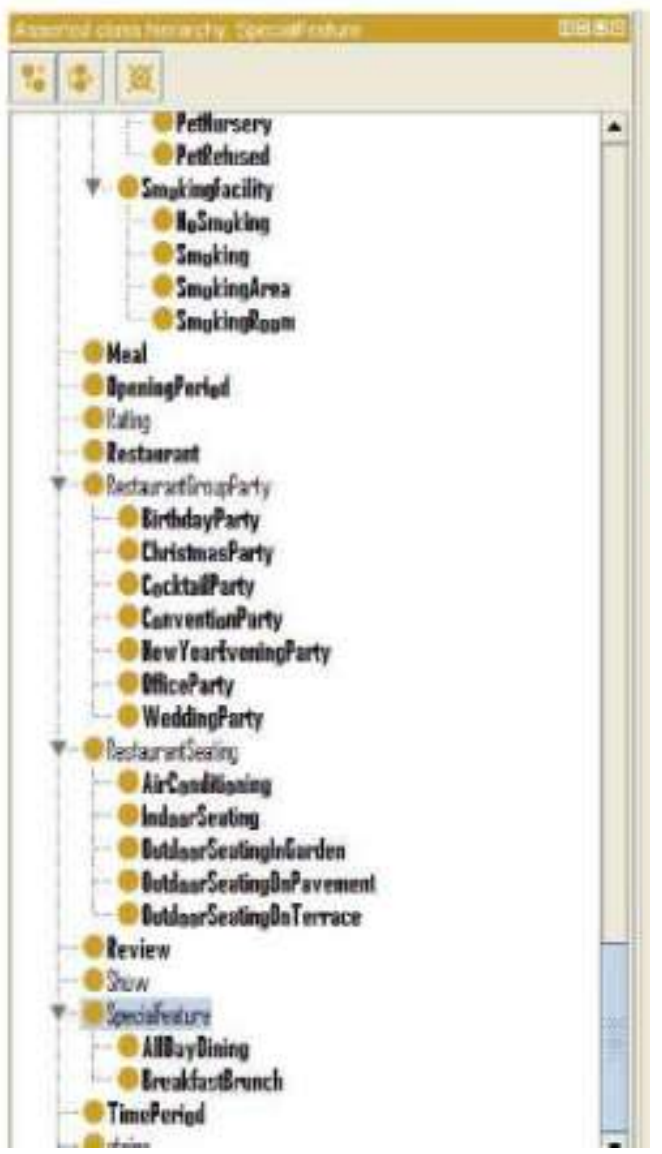


This ontology shows disabled facility, elevator exist, wheel chair access, wheel chair toilet exist \&also parking facility can be viewed as indoor parking, outdoor parking, private parking, public parking, payment facility is specified, wheather card accepted. whether pet facility exists, petfood, petnursery, smoking facility available, smoking room exists, smoking area exists, rating and restaurant group party \&restaurant seating. In restaurant group party, birthday party can be arranged, Christmas party, wedding party, new year party. In restaurant seating, air conditioning is available, indoor seating, outdoor seating \&also some special features are all day dining, breakfast brunch, time period and all these details can be viewed. e web using semantic markup derived from the designed OWL ontology. The dining data were encoded in the RDF format, which can be used to instantiate the ontology. .

\section{Conclusion}

In this paper ,It is presented as results of some experiments performed in order to evaluate retrieval efficiency of an ontology-based approach For example with any technique for assigning concepts from ontology to a query, e.g. based on manual assignment or based on synonyms to query terms, making use of verbnet. Our work will be focused on further enhancement of ontology-based retrieval mechanism using more sophisticated inference mechanism for finding similar concepts to given query. Thus it is implemented in Cloud platform.

\section{References}

[1]. Ontology-based Information Retrieval Jan Paralic 'Department of Cybernetics and AI, Technical University of Kosice,Letna 9, 040 11 Kosice, Slovakia jan.paralic@tuke.sk, Ivan Kostial

[2]. Department of Cybernetics and AI, Technical University of Kosice,Letna 9, 04011 Kosice, Slovakia ivan.kostial@ @uke.sk

[3]. Abecker A., Bernardi A. Hinkelmann K. Kühn, O. \& Sintek M. (1998): Toward a Technology for Organizational Memories, IEEE Intelligent Systems, 13, May/June,p.40-48.

[4]. Volume 3, Issue 10, October 2013 ISSN: 2277 128X International Journal of Advanced Research in Computer Science and Software Engineering

[5]. Ontology Based Information Retrieval - An Analysis Sakthi Murugan R, P. Shanthi Bala Dr. G. Aghila

[6]. Dept. of Computer Science, Dept of Computer Science \& Engg., Pondicherry University, National Institute of Technology (NIT), Puducherry, India. Karaikal, India.

[7]. Qian Gao, "Similarity Matching Algorithm for Ontology-Based Semantic Information Retrieval Model," Proceedings of the 10th World Congress on Intelligent Control and Automation, Beijing, China, p. 758-763.

[8]. HU Jun, LI Zhi-lu and GUAN Chun, “A Method of Rough Ontology-based Information Retrieval,” IEEE International Conference on Granular Computing, 2008, p. 296-299.

[9]. Rashmi Chauhan, Rayan Goudar, Robin Sharma and Atul Chauhan, "Domain Ontology based Semantic Search for Efficient Information Retrieval through Automatic Query Expansion," International Conference on Intelligent Systems and Signal Processing (ISSP), 2013, p. 397-402.

[10]. Jibran Mustafa, Sharifullah Khan and Khalid Latif, "Ontology Based Semantic Information Retrieval," 4th

[11]. International IEEE Conference Intelligent Systems, vol. 3, 2008, p. 14-19.

[12]. Miriam Fernández, Iván Cantador, Vanesa López, David Vallet, Pablo Castells and Enrico Motta, "Semantically enhanced Information Retrieval: An Ontology-based approach," JWS special issue on Semantic Search, vol. 9-4, 2011, p. 434-452

[13]. Sheng Qiuyan and Ying Guisheng, "Measuring Semantic Similarity in Ontology and Its Application in Information Retrieval," International Conference of Soft Computing and Pattern Recognition, 2011, p. 27-32.

[14]. Ying Chen, Wenping Guo and Xiaoming Zhao, "A semantic Based Information Retrieval Model for Blog," Third International Symposium on Electronic Commerce and Security, 2010, p. 257-260.

[15]. SIGIR 2007 Proceedings Session 16: Learning to Rank II, AdaRank: A Boosting Algorithm for Information Retrieval Jun Xu Microsoft Research Asia No. 49 Zhichun Road, Haidian Distinct Beijing, China 100080 junxu@microsoft.com Hang Li Microsoft Research Asia No. 49 Zhichun Road, Haidian Distinct Beijing, China 100080 hangli @ microsoft.com 